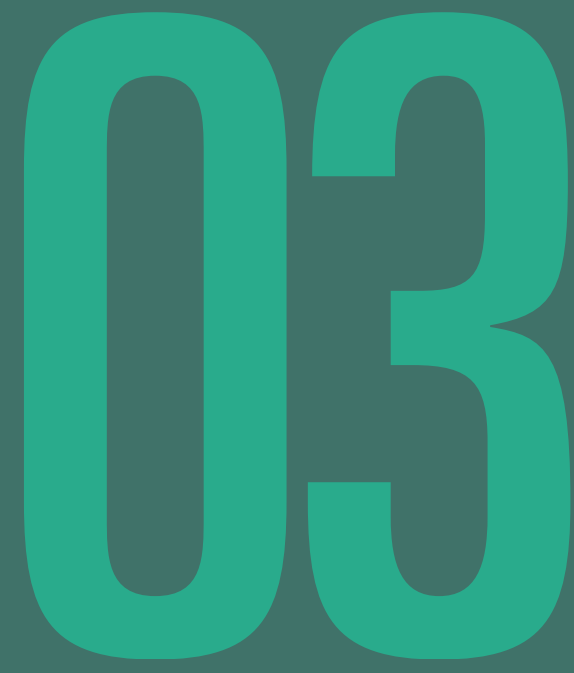

\title{
CONCEPTO Y LUGAR, LA OBRA DEL ARQUITECTO PORTUGUÉS ÁLVARO SIZA
}

\section{Concept and place, the Works of portuguese architect Álvaro Siza}

Cuauhtémoc Robles Cairo

cuauhtemoc@uabc.edu.mx

https://orcid.org/0000-0003-2452-4960

Maestro en arquitectura, profesor investigador de

la Facultad de Arquitectura y Diseño, UABC

Dra. Claudia Marcela Calderón Aguilera

claudiacalderon@uabc.edu.mx

https://orcid.org/0000-0001-7844-2800

Doctora en arquitectura, profesora investigadora de la Facultad de Ingeniería, Arquitectura y Diseño, UABC 
Palabras clave: onceptualización arquitectónica, contexto, Álvaro Siza, arquitectura portuguesa

Keyword: Architectural conceptualization, Context, Alvaro Siza, Portuguese architecture

Resumen:

En el presente artículo se expone una valoración de las primeras obras del arquitecto portugués Álvaro Siza Vieira, desde la perspectiva de la definición conceptual en relación con las características del contexto físico en donde se encuentran emplazadas. Constituye una invitación a reflexionar sobre uno los principios inherentes al objeto arquitectónico, que es la relación de edificio con el lugar y se cuestiona como punto de partida el uso desproporcionado que se tiene, en el medio académico-estudiantil principalmente, de la conceptualización análoga que evoca formas innovadoras sin sustento, bajo el amparo de la subjetividad y la creatividad individual, pero sin el compromiso con el entorno preexistente. Se presenta la descripción de cuatro edificios emblemáticos: las Piscinas des mares, el restaurante Boa Nova, la Escuela de Arquitectura y el Centro Gallego y se concluye con una reflexión sobre la obra del arquitecto y con una serie de tópicos que permita a los estudiantes valorar los temas de análisis como un camino hacia una conceptualización vinculada al lugar.
Abstract:

This article presents an assessment of the first works of the portuguese architect Alvaro Siza Vieria, from the perpective of the conceptual definition of projects in relation to the characteristics of the physical context where they are located. It is also an invitation to reflect on the inherent principles to the architectural object, such as the relationship of the building with the context. It is proposed as a starting point the wear that is had in the student-academic enviroment on the analogous conceptualization that evoques creative forms without a support, under the protection of individual subjectivity and creativity. The conceptual description of four emblematic buildings analyzed is presented: the Piscinas des mares, the Boa Nova restaurant, The Oporto's Architecture School and the Galician Center and concludes with a reflection on the architec's works and with a thematic instrument that facilitates the students to assess the specific topics as a path towards a conceptualization linked to the place. 
Cuauhtémoc Robles Cairo

Claudia Marcela Calderón Aguilera

\section{INTRODUCCIÓN}

as definiciones conceptuales en el ejercicio del diseño arquitectónico en el ámbito académico con frecuencia recurren en grado excesivo a la conceptualización análoga, que establece relaciones de semejanza entre dos cosas distintas, partiendo de la representación de un objeto. Suele ser un recurso creativo efectivo instalado en la subjetividad del estudiante, en donde la valoración por parte del profesor reside en la afinidad —el agrado-que este tenga con la idea propuesta. Un concepto análogo en el proceso creativo inicial toma como punto de partida la representación de una forma o la función de un objeto y lo traslada a la propuesta arquitectónica, en el entendido de que la imagen es solo un pretexto creativo sin grandes fundamentos; aunque no se tenga la pretensión de copiar — literalmente- la forma del objeto base, lo que resultaría prácticamente imposible cuando en el proceso de composición arquitectónica deben coincidir el concepto, los objetivos de diseño, el análisis del lugar y el programa arquitectónico. Aun así, la esencia de las formas análogas y sus contornos ha quedado plasmada recurrentemente en la arqui- 
tectura contemporánea, donde el tema de la conceptualización ha tomado gran relevancia en las últimas décadas, incluso dejando parcialmente de lado postulados añejos de la arquitectura como es la vinculación del edificio con el terreno que lo acoge.

En la conceptualización análoga se parte de imágenes y se busca establecer relaciones creativas del objeto imaginado sin la necesaria conjunción con el problema de diseño para, después de un proceso de exploración, descifrar las relaciones fortuitas que puedan aportar al desarrollo del proyecto. Este camino tiene sustento en el método sinéctico del psicólogo e inventor estadounidense William J.J. Gordon donde se plantea la resolución de problemas a partir de la conjunción de cosas distintas (Muñoz, 2019, p.52). Y aunque la creatividad es sin duda una parte fundamental del objeto arquitectónico porque conforma parte del pensamiento simbólico, resulta fundamental no abandonar las ideas y objetivos de diseño generados a partir de la comprensión del lugar; la imaginación como soporte creativo de ideas y la lectura perceptual del sitio aunada a la razón como sustento para la integración de un proceso, de una estrategia proyectual. Es decir, establecer un equilibrio entre en mundo subjetivo y objetivo del pensamiento y sentar la base del proceso en conceptos consistentes y fundamentados, como resulta evidente en la obra del arquitecto portugués Álvaro Siza Vieira, objeto de este estudio.

La arquitectura de Álvaro Siza ${ }^{1}$ constituye un ejemplo relevante disociado del protagonismo formal análogo antes aludido, que apuesta en cambio a la valoración y apropiación de las características y posibilidades de un lugar como principio generador del concepto de un proyecto. La lectura del entorno, ya sea natural, urbano o mixto, se prioriza sobre preconcepciones formales y plásticas y encuentra así los recursos creativos vinculados a la identidad del sitio. Un símil frecuentemente establecido con la obra de Siza es el mismo Alvar Alto (1898-1976), arquitecto finlandés asociado al movimiento moderno, quien, sin despegarse del rigor funcional que imponen los requerimientos del programa arquitectónico, supo implantar un lenguaje denominado "organicista"

1 Álvaro Joaquim Siza Vieira nació en Matosinhos, Portugal en 1933; egresado de la Universidad de Oporto, después de una larga vida profesional recibió el premio Pritzker de arquitectura en 1992 
con planteamientos libres más dialogantes con la naturaleza de su entorno. En él, el contorno del terreno, la iluminación y ventilación natural, la orientación heliotérmica, las perspectivas del paisaje y los materiales y técnicas locales crean una conjunción armónica entre usuario, edificio y entorno paisajístico (Baborsky, 2001, p.18).

\section{ANTECEDENTES}

El arquitecto portugués Álvaro Siza Vieira nació en 1933 en Matosinhos, Oporto, en donde trascurrió su infancia, juventud y estudios profesionales; aquí mismo inició su carrera profesional y desarrolló una obra profusa antes de ser conocido internacionalmente. Siza describe sus primeros años de vida como tranquilos y apacibles en el entorno de esta región costera portuguesa, y en su formación menciona como fundamental los viajes familiares, donde las visitas a museos resultaban imprescindibles. Como estudiante de arquitectura en la Escuela de Bellas Artes de Oporto -ESBAP— $(1949-1955)^{2}$, Siza entró en contacto con el arquitecto Fernando Távora (1923-2005), su profesor, con quien colaboró después profesionalmente entre 1955 y 1958. Távora fue un influyente arquitecto portugués, coautor del libro Arquitectura popular em Portugal publicado en 1961, esta obra fue determinante en la definición de una arquitectura portuguesa moderna con una connotación regional, popular y en donde la comprensión del paisaje es un elemento definitivo en la resolución proyectual (Fleck,1999, p.23). A principios de la década de los setenta la obra de Siza empezó a trascender en algunas publicaciones europeas, y en estas primeras lecturas se reconoce al lugar — al contexto- como una noción central de su arquitectura, en un tiempo histórico de revisión de la arquitectura moderna que tuvo comienzo tras la Segunda Guerra Mundial (Illescas, 2017, p.22).

Derivado del cuestionamiento al movimiento moderno como lenguaje unitario -internacional— surgió el regionalismo crítico en la arquitectura, que puso en duda la ausencia de identidad de un estilo internacional y retomó y reinterpretó los valores locales adaptados a las nuevas necesidades en la segunda mitad del siglo XX. Para Frampton ${ }^{3}$, el regionalismo crítico no significó un

2 Escuela de Bellas Artes de Oporto.

3 Kenneth Brian Frampton (1930) Influyente arquitecto y critico de arquitectura de origen británico, egresado de la Architectural Association de Londres. Autor de Towards a Critical Regionalism, 1983. 
abandono de la arquitectura moderna, porque su valor progresivo permitía la incorporación del contexto geográfico en donde se sitúa un edificio (Frampton, 1983). Aunque referido con frecuencia al mencionado regionalismo crítico y al contextualismo contemporáneo, Siza en realidad aborda conceptualmente cada obra como una oportunidad única, sin la pretensión de crear un lenguaje personal y con la aproximación lógica de la lectura del entorno, y por tanto con la frescura que brinda cada emplazamiento en particular. La apropiación local en lo que concierne al estudio de tipologías y materiales no tiene la finalidad de repetir modelos sino de crear actualizaciones, considerando a la actualización como un mecanismo progresivo (Gómez, Gómez y Godoy, 2011, p.128).

Después de una serie de proyectos en la región de Oporto, Siza realizó en 1986 la Agencia del Banco Borges e Irmâo, en Vila do Conde, una población costera en la desembocadura del río Ave, ésta fue la primera obra que lo posicionó internacionalmente después de la obtención del premio europeo de arquitectura Mies van der Rohe en 1988 (Catarratala, 2003, p.12). El edificio se encuentra en un contexto con una fuerte inercia histórica, frente a una arboleda antigua y la iglesia Matriz, rodeado de casas señoriales y en consonancia con el entorno existente adoptó la escala urbana de las construcciones vecinas. Su sencillez formal y material por medio de muros lisos y blancos revelan la búsqueda de una saludable integración mas no una mimesis; incluso el hecho de que el edificio asome hacia la calle mediante un vértice y no a través de un plano frontal, alude a esta condición de convivir con los edificios vecinos sin protagonismos excesivos. A partir del Banco en Vila do Conde, se abrió un amplio camino de reconocimiento internacional que lo llevó después de una prolífica trayectoria de cuatro décadas a recibir el premio Pritzker de arquitectura en 1992, el más prestigiado galardón de reconocimiento a la obra de un arquitecto a nivel mundial, premio concedido por su «respeto a las tradiciones de su Portugal nativo» y su «respeto por el contexto» (The Hyatt Foundation, 2021).

\section{OBJETO DE ESTUDIO}

Se seleccionaron cuatro obras del arquitecto Álvaro Siza desarrolladas durante sus primeros treinta años de carrera, tres de ellas ubicadas en el contexto local del Gran Oporto -Porto- y una externa, en Santiago de Compostela, 
Galicia, pero localizada también en el mismo contexto geográfico que es el noroeste de la península Ibérica. Oporto —el Puerto— se ubica en la ribera derecha del río Duero, geográficamente al norte de la costa portuguesa en la desembocadura del afluente mencionado, en la provincia denominada como Región Norte de Portugal. Esta tiene un clima benigno de tipo marítimo templado $^{4}$ con temperaturas que no llegan a superar los 3

$0^{\circ} \mathrm{C}$ en verano y que no bajan menos de $1^{\circ} \mathrm{C}$ en invierno, cuando se presenta la temporada lluviosa. El verano suele ser seco y despejado, favorecido con los vientos del océano Atlántico.

En cuanto a su historia, aunque se han encontrado vestigios arqueológicos que evidencian una ocupación celta y griega — villa de Cale—, la ciudad fue reconocida en el siglo IV como un importante puerto comercial suevo denominada entonces como Portus Cale, del cual deriva la palabra Portugal. Durante el imperio romano funcionó como enclave fundamental entre Lisboa y Braga; y fue un sitio fundamental donde se fraguó la guerra de Reconquista, tras la ocupación musulmana. El comercio del vino, particularmente con Inglaterra, definió en buena medida su carácter internacional y progresista. Oporto es la segunda ciudad más grande de Portugal, la zona conurbada conformada por el Gran Oporto aloja una población que ronda en los tres millones de personas en 2020 y su Centro Histórico fue declarado patrimonio de la humanidad por la UNESCO en 1996 (Bonamusa, 2011, p.19).

Santiago de Compostela tuvo un origen celta con el nombre de Libredom, posteriormente fue conocida como Castreña por los romanos y con la llegada del cristianismo adquirió el nombre de Compostela, que al parecer deriva de la combinación de las palabras Campus Stellae o Campo de la Estrella en alusión, de acuerdo con la leyenda, a la estrella que alumbró el camino hacia el lugar en donde se encontraban los restos del apóstol Santiago, El Mayor. La ciudad ha tenido un papel relevante como destino importante de peregrinación cristiana, como culminación de El Camino de Santiago. En la actualidad cientos 4 Denominado Clima mediterráneo con influencia oceánica (Csb) según la clasificación de Köppen-Geiger. 
de personas realizan un recorrido por esta ruta, pernoctando en posadas que dieron origen a buen número de pueblos que bordean el camino, es también un trayecto importante del románico español.

Compostela se localiza al noroeste de España en la región de Galicia, además del hecho histórico religioso mencionado, es conocida por su famosa catedral barroca y por contar con una universidad de más de 500 años de antigüedad que le imprime a la ciudad notable vida estudiantil.

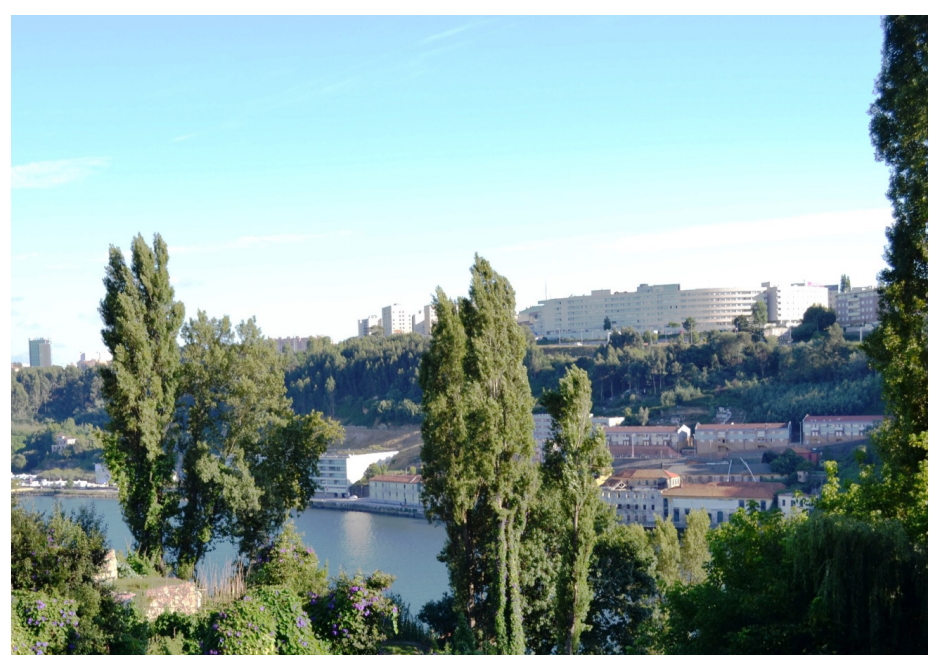

Figura 1, Oporto, Portugal. Vista hacia el estuario del río Duero, desde la Escuela de Arquitectura. Autor: Calderón Aguilera, Claudia, 2015.

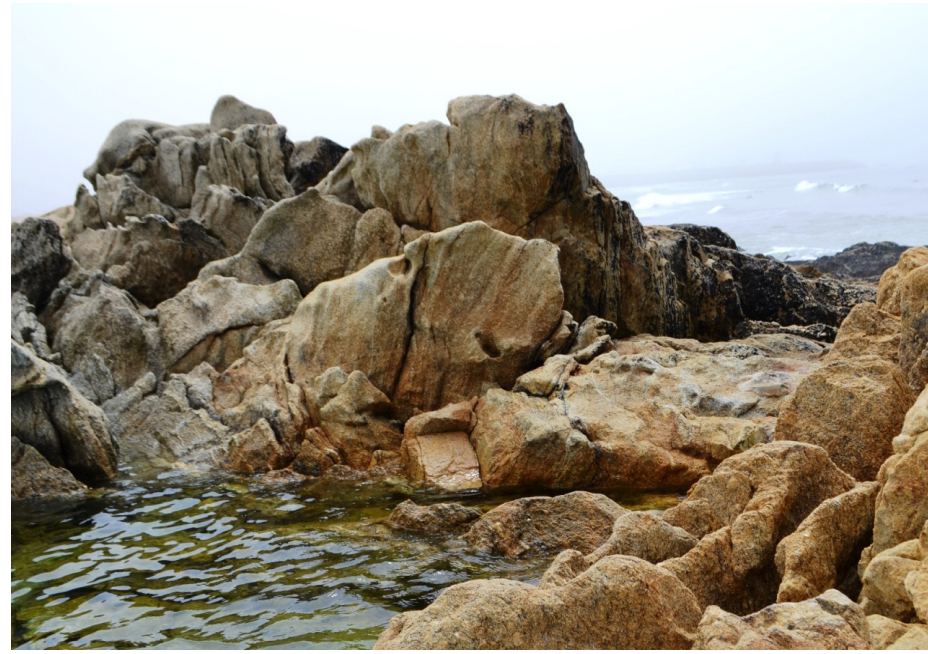

Figura 2, Matosinhos, formaciones rocosas en Leca de Palmeira. Autor: Calderón Aguilera, Claudia, 2015. 


\section{Obras de referencia:}

-Piscina de Marés, Matosinhos, Gran Oporto (1961-1966)

-Restaurante Boa Nova, Leca de Palmeira, Matosinhos, Gran Oporto (1963)

-Escuela de Arquitectura de Oporto, (1985-1996)

-Centro Gallego de Arte Contemporáneo CGAC, Santiago de Compostela, Galicia (1993)

\section{1) PISCINA DES MARÉS, MATOSINHOS, GRAN OPORTO (1961-1966)}

El paisaje costero de Portugal fuertemente erosionado por los vientos del Atlántico, tiene una connotación escarpada con interesantes formaciones geológicas, que en un par de proyectos de Siza definen el concepto de diseño, como en las Piscinas de Mar. Se trata de dos albercas de agua salada emplazadas junto a la costa marítima, la de mayores dimensiones para adultos y otra menor para niños. En estas, Siza aprovechó la existencia de grandes macizos rocosos entre los cuales creó muros de contención de concreto para delimitar las piscinas, potenciando así los espacios naturalmente conformados y aprovechables para crear los embalses.

El proyecto recrea como concepto un estanque de apariencia natural, aparentemente alimentado con el vaivén de las mareas, aunque cuenta con un sistema mecánico de renovación del agua. En la alberca para adultos, los muros de concreto tienen un ancho suficiente para que las personas puedan caminar, sentarse o recostarse en ellos a tomar el sol y los baños-vestidores y cafetería se encuentran parcialmente bajo el nivel de la calle, liberando las vistas desde la avenida hacia el mar. En tanto, las cubiertas de madera de roble oscuro recubiertas con láminas de cobre oxidado por la humedad marina le confieren una apariencia mimética, ejercida por el desgaste de los elementos naturales sobre el edificio, evidenciando un claro sentido evolutivo marcado por el efecto de la naturaleza.

El proyecto de las piscinas se adapta al paisaje y no al revés, bañarse en ellas es incursionar en las aguas saladas del Atlántico que suelen presentar súbitas y violentas irrupciones como lo precisó el mismo Siza en un momento del 
proceso conceptual. El proyecto se sitúa en los límites entre el paisaje natural y urbano, separados apenas por una avenida costera. Su naturaleza pétrea expuesta a la erosión obedece más a los confines de lo marítimo; y, aunque acotar los límites constituye un primer paso en la intervención de un territorio, la línea de playa se presenta como un límite difuso y temporal, como son límites del proyecto, se transita sobre este en diferentes direcciones. Su emplazamiento y forma se oponen a la estructura confinada — delimitada- que suele presentar generalmente la arquitectura.

En este sentido, Fleck (1999) cita a Álvaro Siza:

Todos los años con las mareas vivas el mar se lleva lo que no es esencial. En ese lugar, un macizo rocoso interrumpe las tres líneas paralelas: el encuentro del mar y el cielo, el de la playa y el mar, y el largo muro de contención de la vía Marginal -bulevar-, y alguien pensó en proteger una depresión formada entre ese macizo y construir unas piscinas de mareas, y deliberó en llamar a un arquitecto. Nada ha cambiado, el edificio del balneario permanece anclado como un barco al muro de la avenida, de ahí no se mueve, aunque con las primeras mareas el mar se llevó un trozo de muro, corrigiendo lo que no estaba bien (p.28).

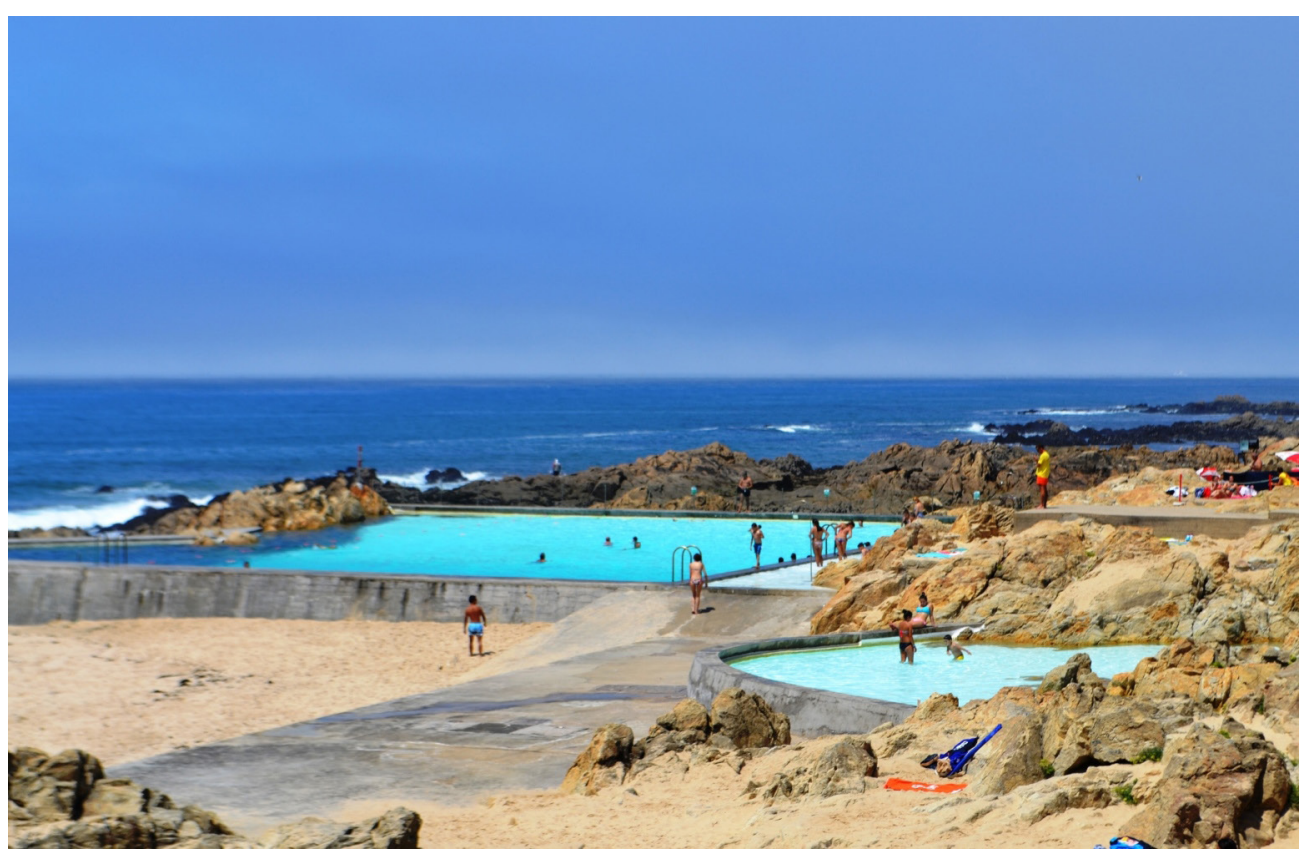

Figura 3. Piscina des Marés, Matosinhos, Portugal. Perspectiva, en primer plano el chapoteadero. Autor: Calderón Aguilera, Claudia, 2015. 


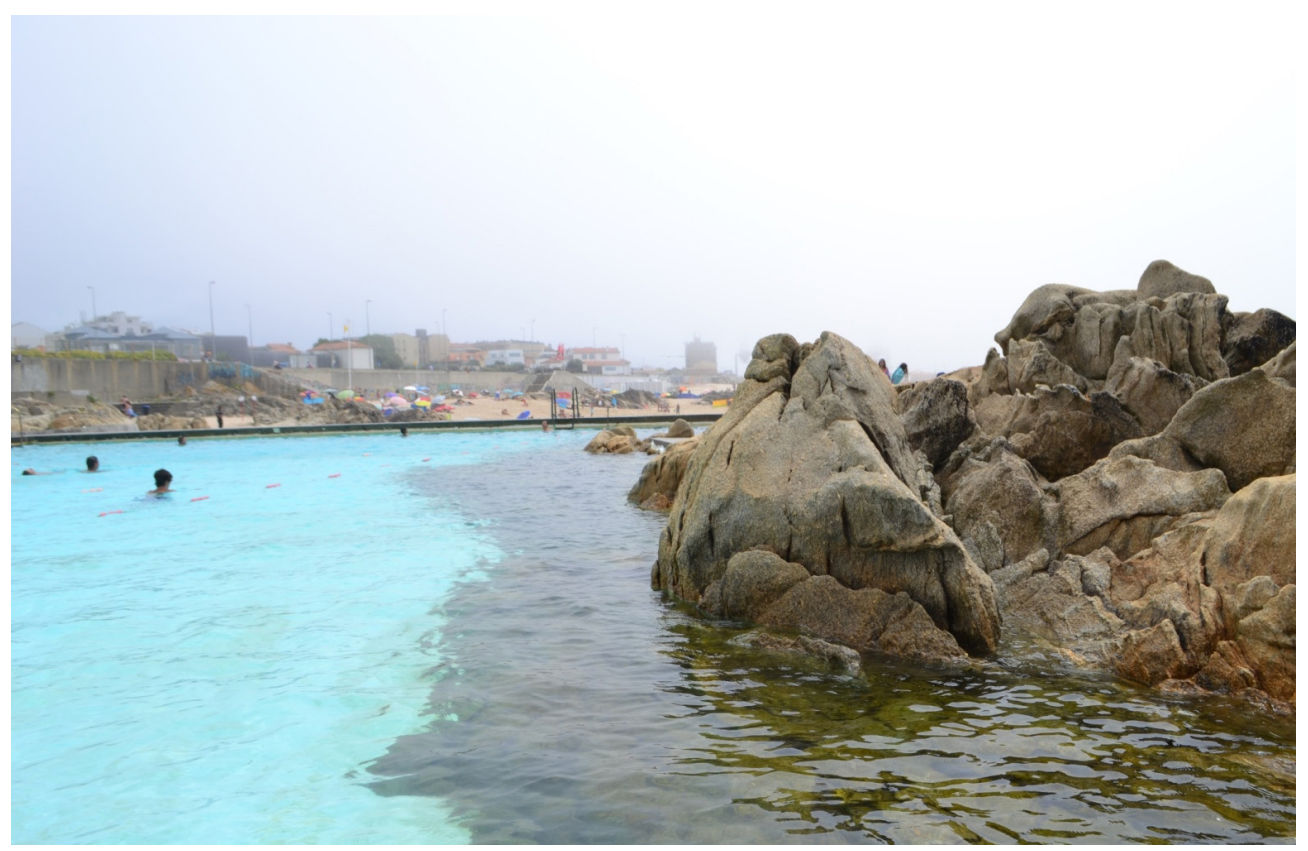

Figura 4. Piscina des Marés, Matosinhos, Portugal. Conjunción entre naturaleza y edificación. Autor: Calderón Aguilera, Clandia, 2015.

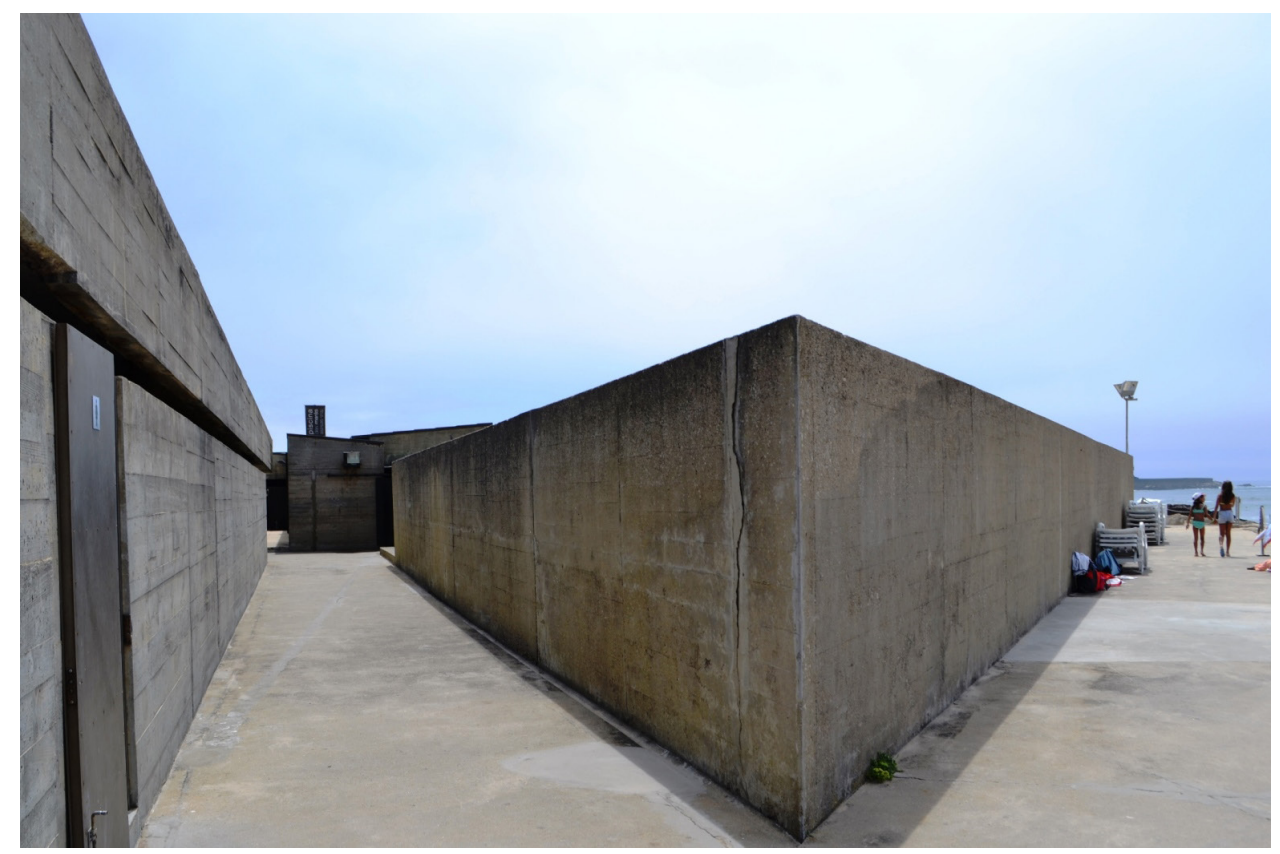

Figura 5. Piscina des Marés, Matosinhos, Portugal. Rampas de acceso y área de baños-sanitarios. Autor: Calderón Aguilera, Claudia, 2015. 


\section{2) RESTAURANTE BOA NOVA, LECA DE PALMEIRA, MATOSINHOS} (1963)

Otro proyecto fuertemente marcado por la topografía costera es el restaurante Boa Nova en Matosinhos, Portugal. Es producto de un concurso convocado en 1958, donde el arquitecto realizó una obra emplazada en un hermoso paraje costero entre un montículo rocoso, lo cual de entrada implicó el reconocimiento de las condiciones meteorológicas, un estudio de la flora y del efecto de las mareas. Siza aprovechó la concavidad natural formada en el montículo para ubicar el edificio, el cual oculta sus muros de concreto aparente entre piedras y resalta la cubierta de teja como el elemento formalmente predominante, su cúspide parece cerrar el perfil de este pequeño cerro rocoso y sus aleros pronunciados no sólo protegen los muros y ventanales, sobre los cuales se refleja el paisaje, sino que además resguardan los encaminamientos exteriores inmediatos del edificio. Los ventanales amplios facilitan una relación visual entre exterior-interior, también permiten iluminar los espacios con luz natural, una constante de su obra.

Además de la topografía accidentada del emplazamiento, de su proximidad a la costa, el proyecto interpreta las condiciones climáticas del lugar, lo que se manifiesta en la orientación del edificio; en la utilización de materiales, como la teja de barro y en los muros de concreto; en la forma de las cubiertas inclinadas; en el tamaño y forma de los vanos de los muros, restringidos hacia la ciudad y abiertos hacia el mar, y particularmente en la incorporación de recursos de integración bioclimática como los pronunciados aleros que generan terrazas intermedias o transitorias entre exterior e interior. En este sentido Campos (2014, p.13.) señala que Siza "conocía bien esta zona rocosa de la costa: la suave inclinación, un suelo desnudo, con grandes rocas junto al mar”. 


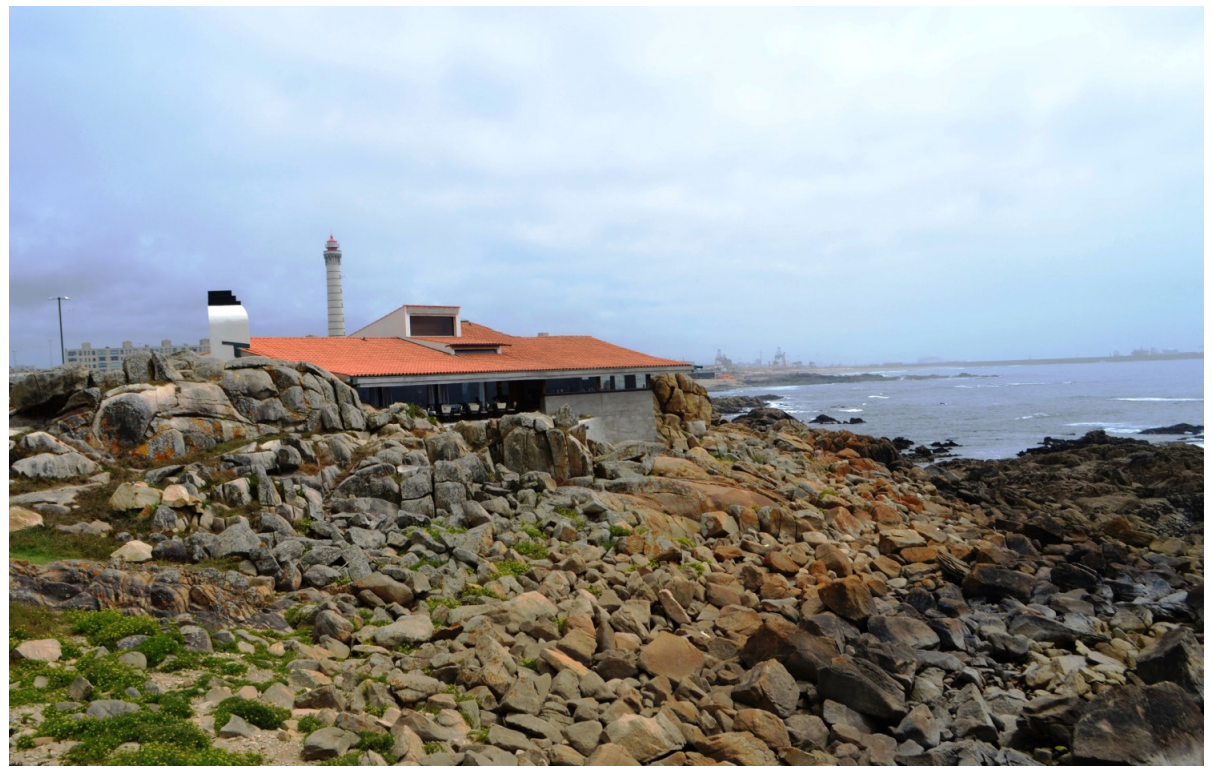

Figura 6. Restaurante Boa Nova, Leca de Palmeira, Matosinhos, Portugal. El restaurante en el montículo rocoso. Autor: Calderón Aguilera, Claudia, 2015.

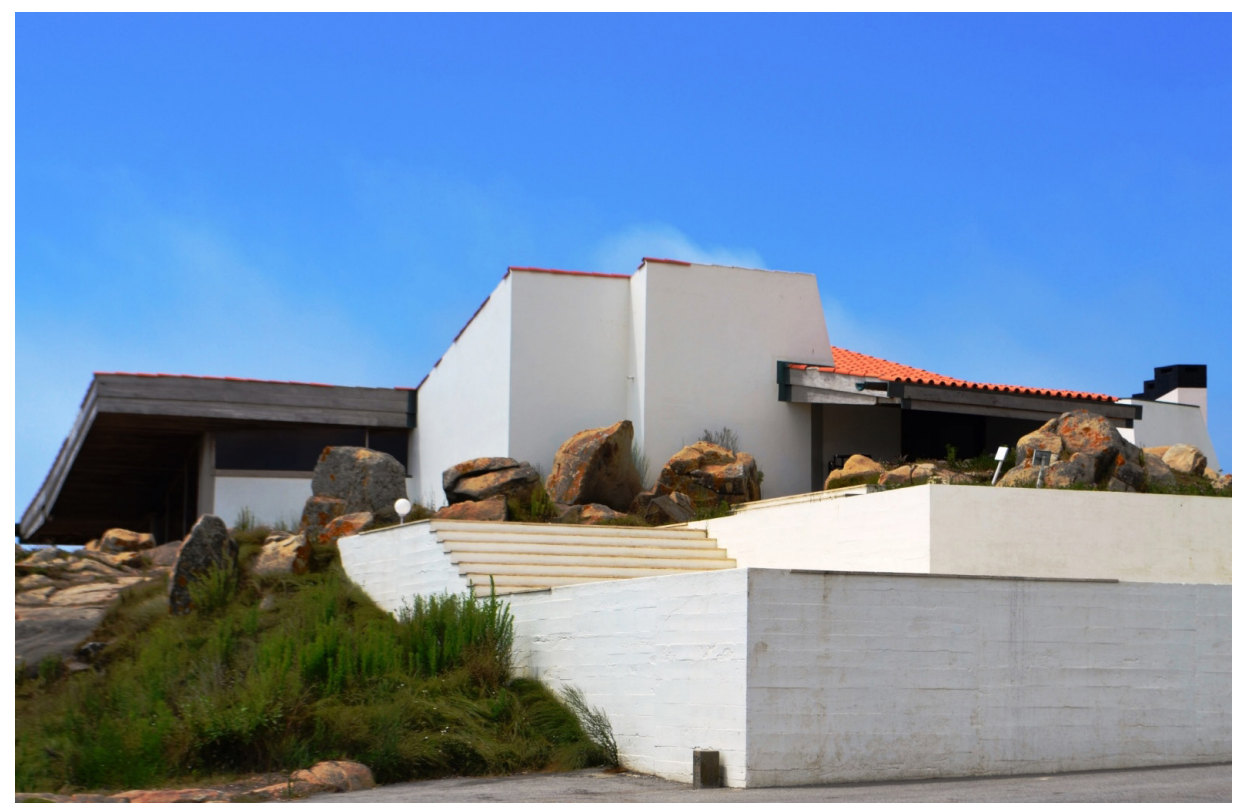

Figura 7. Restaurante Boa Nova, Leca de Palmeira, Matosinhos, Portugal. Fachada desde el estacionamiento. Autor: Calderón Aguilera, Claudia, 2015 


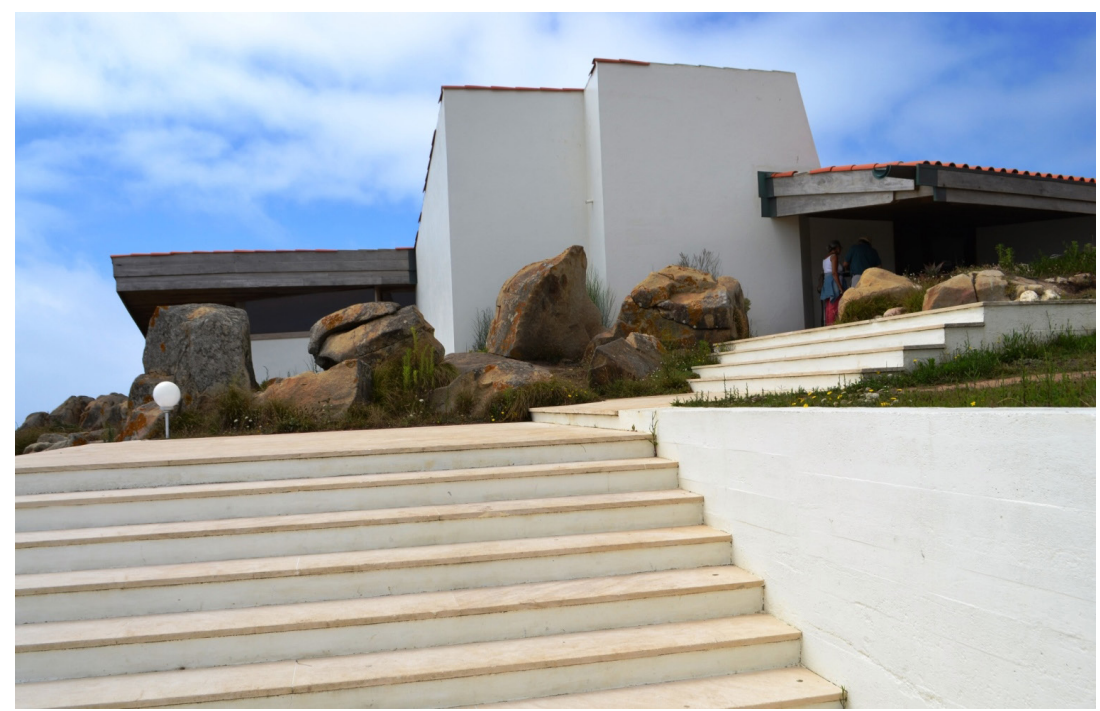

Figura 8. Restaurante Boa Nova, Leca de Palmeira, Matosinhos, Portugal. Escalinata y acceso. Autor: Calderón Aguilera, Claudia, 2015.

\section{3) ESCUELA DE ARQUITECTURA DE OPORTO, (1985-1996)}

En un lugar excepcional sobre una colina arbolada en el estuario del río Duero en Oporto, Siza realizó el proyecto de la Escuela de Arquitectura. Conceptualmente la solución emplea un esquema compositivo que abre las vistas hacia el paisaje desde el centro del predio, se trata de un conjunto de ocho edificios dispuestos en torno a una plaza semi triangular condicionada por la forma del terreno. Hacia el norte de esta se dispusieron las oficinas administrativas, auditorio, galería y biblioteca; mientras que, hacia el lado sur, que corresponde con el perfil de la colina, dispuso cuatro pabellones de cinco niveles cada uno que albergan aulas y talleres; entre éstos, el vacío favorece la permeabilidad visual hacia el estuario. Los edificios se comunican entre sí a través de una serie de pasajes subterráneos, liberando con ellos la materialidad de los pabellones. Los pasillos interiores y exteriores, las rampas y escaleras presentan el protagonismo de la interacción personal que el arquitecto quiso lograr en espacios de encuentro, más allá de la función utilitaria de transitar en ellos.

El acceso al conjunto ocurre por el costado poniente del terreno a través de una rampa suave recubierta de césped, que por la forma triangular del predio acentúa la perspectiva de la plaza. Las elevaciones volumétricas independientes pintadas de color blanco con sus sencillos ventanales horizontales, 
muy lecorbusianos, sobresalen sobre el paisaje verde del bosque. Desde los talleres y aulas se busca crear una conexión visual con la ciudad; los vanos de ventanas que se perciben sin un patrón en las fachadas, en realidad enmarcan vistas específicas que el arquitecto quiso destacar.

El edificio por su tipo de actividades y emplazamiento logra equilibrar las transiciones entre tres niveles de escala: el espacio íntimo de las aulas y oficinas resuelto a una escala doméstica, derivado de las dimensiones y funciones del cuerpo humano; el segundo orden de escala que corresponde al espacio público contenido en la plaza que articula el conjunto y que parcialmente se abre hacia el entorno, y finalmente la escala urbana en donde el conjunto se sitúa, visto desde el estuario como un hito urbano, a decir como una acrópolis blanca que emerge sobre el paisaje boscoso y que además simbólicamente aloja a una institución educativa.

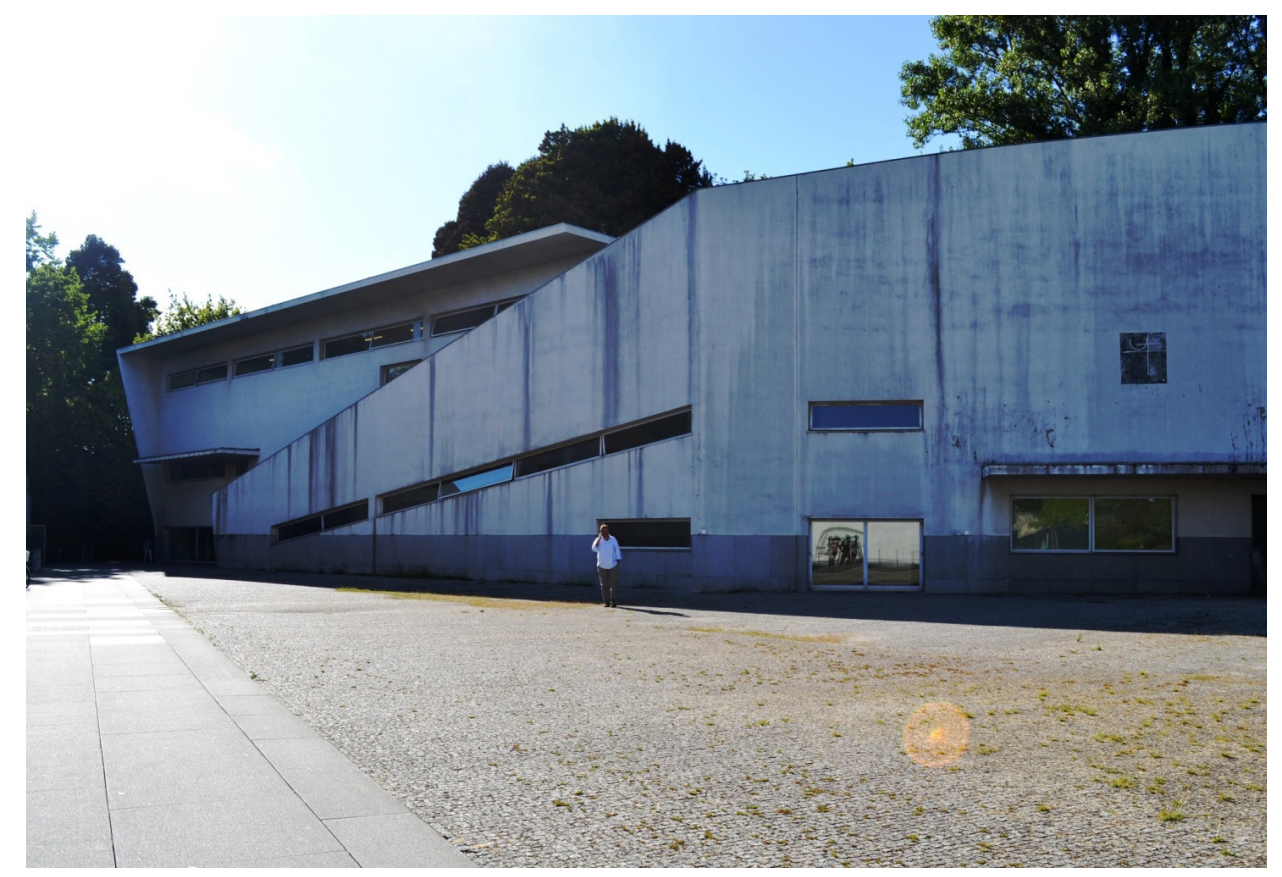

Figura 9. Escuela de Arquitectura de Oporto, Portugal. Edificios de aulas y talleres Autor: Calderón Aguilera, Claudia, 2015. 


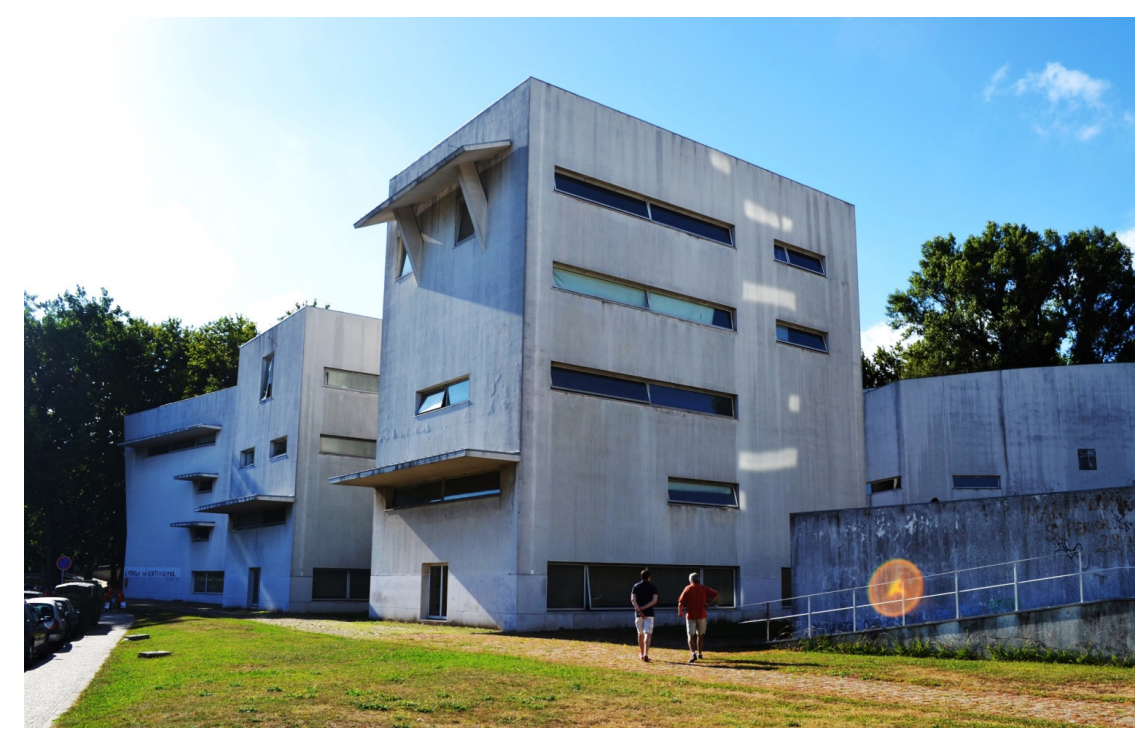

Figura 10. Escuela de Arquitectura de Oporto, Portugal. Patio central y vista hacia el acceso. Autor: Calderón Aguilera, Claudia, 2015.

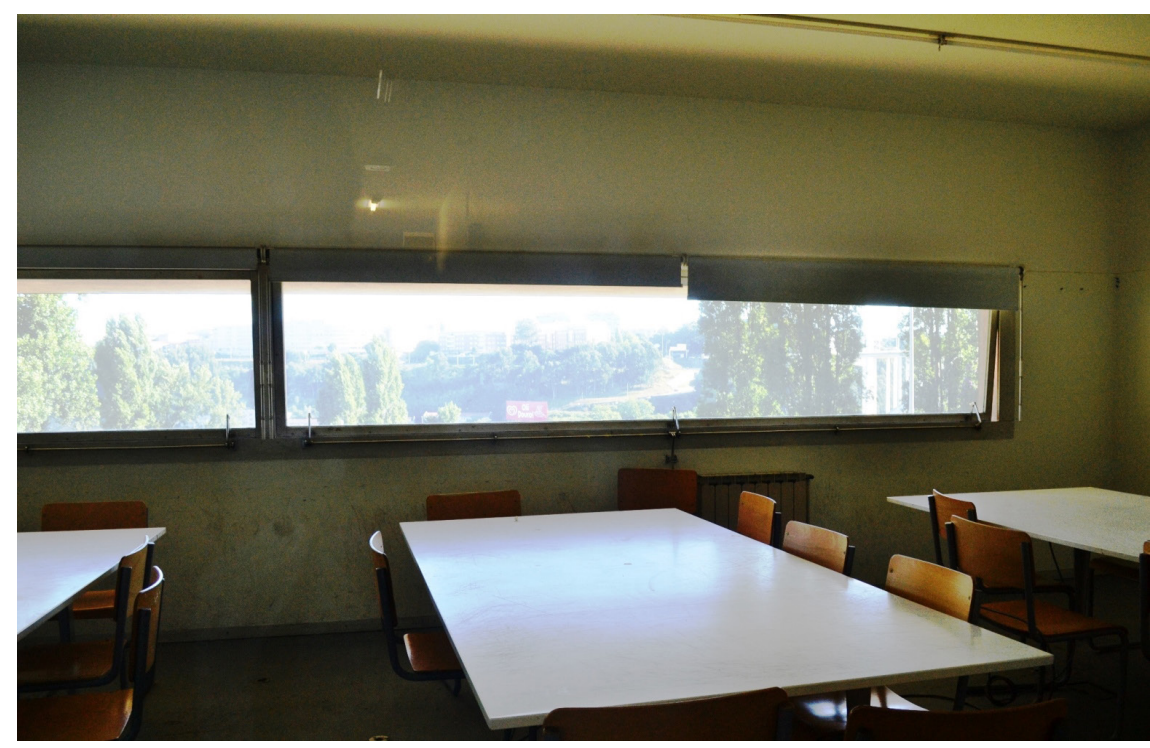

Figura 11. Escuela de Arquitectura de Oporto, Portugal. Vista hacia el Duero desde aula. Autor: Calderón Aguilera, Claudia, 2015.

\section{4) CENTRO GALLEGO DE ARTE CONTEMPORÁNEO, CGAC, (1993)}

El terreno en donde se encuentra el Centro Gallego de Arte Contemporáneo se localiza en la periferia del Centro Histórico, tiene forma triangular, pendiente escalonada y está contenido entre vialidades angostas en tres de sus lados. En otro de sus costados una estrecha calle peatonal lo separa del convento de Santo Domingo de Bonaval, construido a finales del siglo XVII, y por tanto la estructura dominante en el lugar por su valor histórico, dimensiones e 
inercia formal, resultante de sus muros de mampostería de piedra oscurecida por el tiempo. Su torre campanario mantuvo su jerarquía en el contexto aún con la presencia del edificio de Siza.

El Centro Gallego ocupa todo el predio alineando sus muros al límite de propiedad, los paramentos sólidos recubiertos de granito le confieren una inercia que dialoga con la presencia del edificio histórico. La fachada de acceso cuenta con una sustracción en el volumen que aloja la rampa de ingreso al edificio, la cual permite solventar las diferencias topográficas en el lugar y entre las calles vecinas, específicamente entre el nivel de la calle y el vestíbulo de acceso. En este también converge la escalinata frontal al convento, se crea así un paso que reúne visual y físicamente las puertas de ambos edificios (Kliczkowski, 2001, p.8). Los muros liberados en la parte inferior, como en el cerramiento del vestíbulo, son recurrentes en las obras de Siza, permiten enmarcar perspectivas, contener el espacio y generar sombras sobre las áreas vestibulares.

La relación entre museo y convento se extiende además a la terraza de exposiciones de donde se tienen espléndidas vistas hacia la ciudad, y particularmente hacia el parque público que antes fueron los huertos del convento. El parque ocupa una posición intermedia entre el terreno que aloja el CGAC y la ciudad. Con el paso de los años el clima húmedo y lluvioso de Santiago de Compostela ha matizado y envejecido el color y aspecto novel de los muros de granito, provocando la presencia de un edificio con forma de prisma matizado ya por los efectos del tiempo. Al interior y de acuerdo con la forma del terreno, un atrio de forma triangular que atraviesa los tres niveles de plantas permite organizar los diversos espacios como salas de exposiciones temporales, salas conferencias, de juntas, seminarios y las oficinas de administración. El atrio a la vez funciona como núcleo de luz hacia el ámbito neutro de las salas de exposiciones, donde también se tienen vistas secuenciadas de la ciudad por medio de ventanales horizontales. 
El Centro Gallego convive con un inmueble de fuerte carácter histórico, en su entorno se encuentran arraigadas estructuras que muestran formas de vida del pasado, o bien que fueron sede de importantes eventos históricos, que a la postre tuvieron repercusiones en un grupo social o en diferentes generaciones. La especificidad histórica del lugar habla de origen y transformación en el tiempo, lo que predispuso al arquitecto hacia la búsqueda de una integración urbana, al crear un ambiente estable en el presente, ordenado a partir de las siguientes reglas básicas: recuperar el paramento de los edificios que conforman la calle, la consideración de la altura de las edificaciones vecinas, la integración cromática con materiales que al envejecer adquirirán la pátina del contexto, pero sobre todo marcando una diferenciación estilística con el edificio del convento, constituido como un hito simbólico.

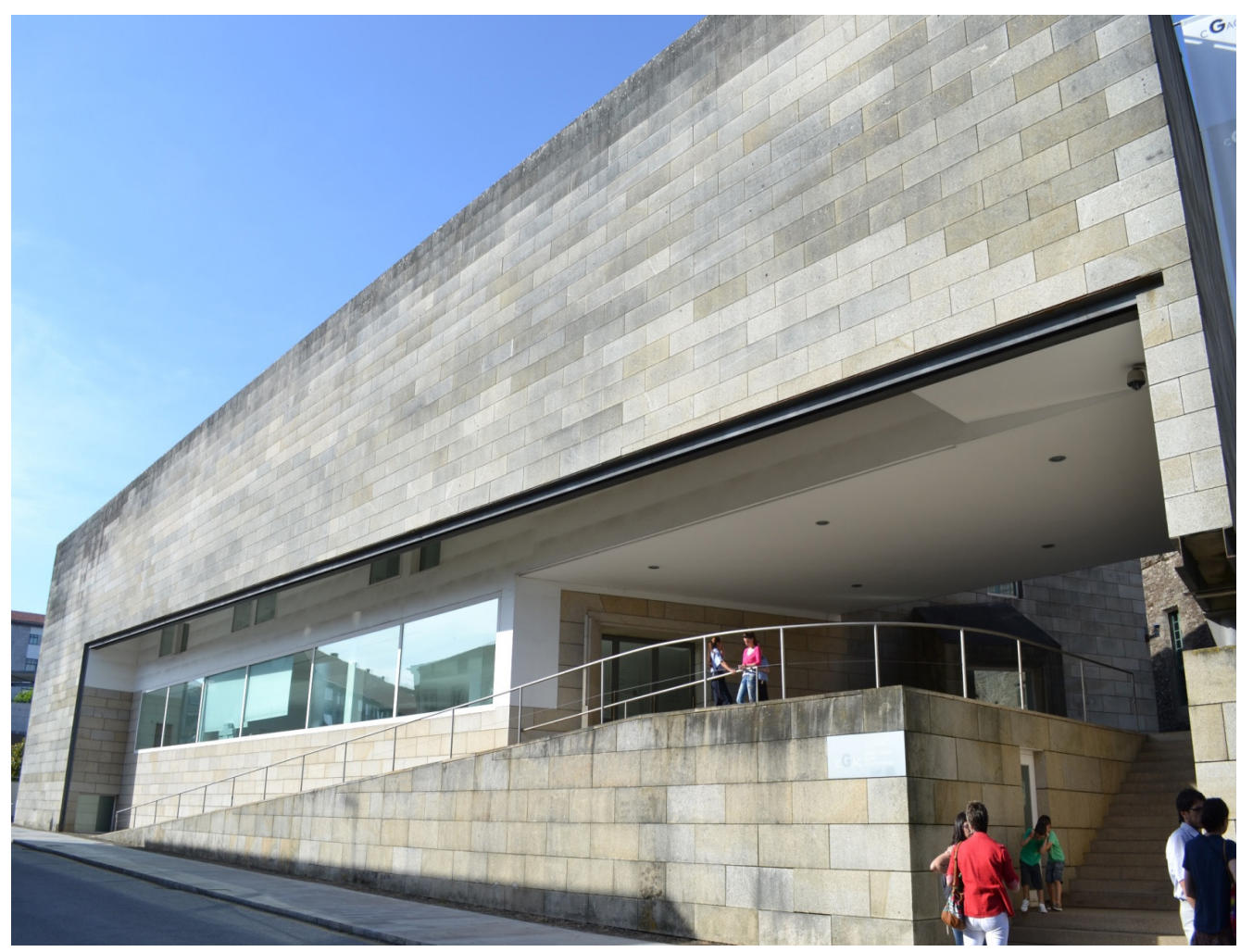

Figura 12. Centro Gallego de Arte Contemporáneo, Santiago de Compostela, España. Fachada principal y acceso. Autor: Calderón Aguilera, Claudia, 2015. 


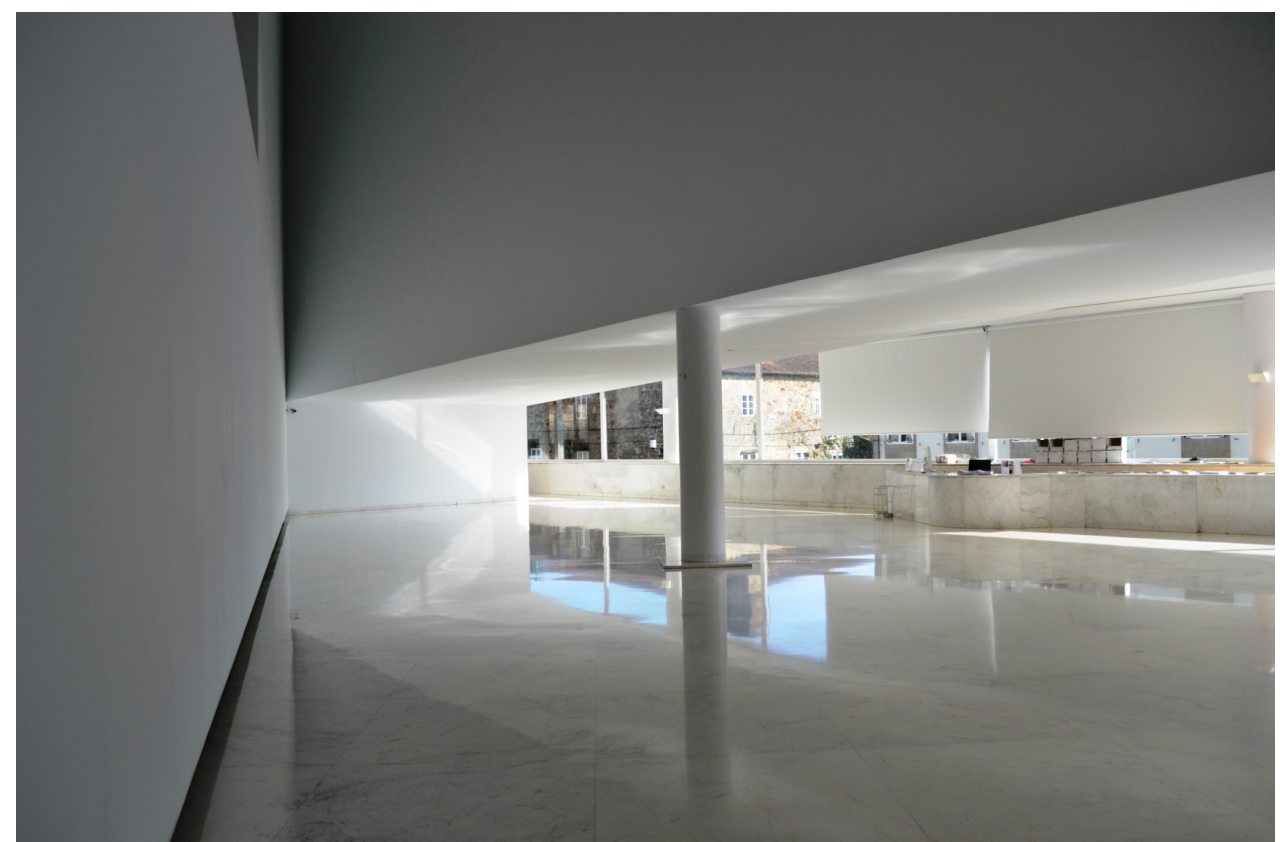

Figura 13. Centro Gallego de Arte Contemporáneo, Santiago de

Compostela España. Atrio interior. Autor: Calderón Aguilera, Claudia, 2015.

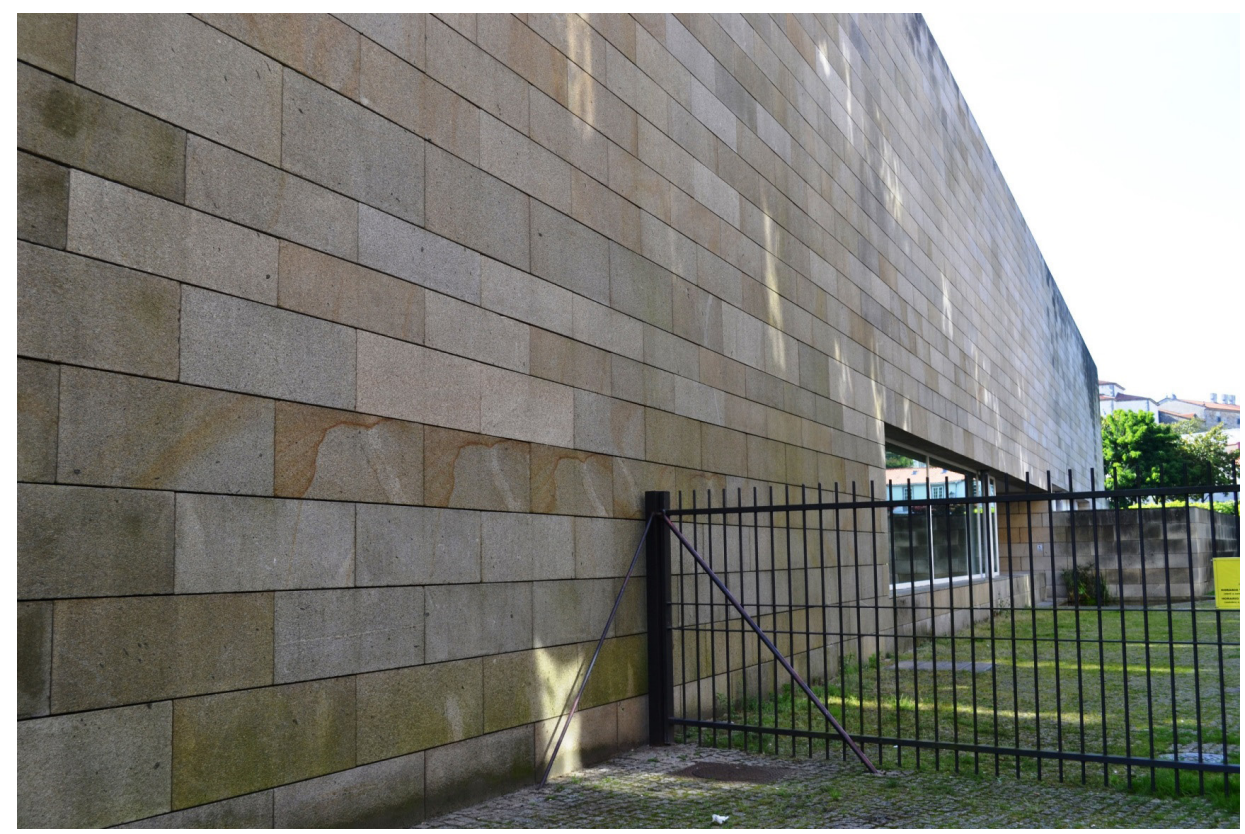

Figura 14. Centro Gallego de Arte Contemporáneo, Santiago de Compostela, España. Fachada posterior hacia el convento. Autor: Calderón Aguilera, Claudia, 2015. 
Tabla 1. Definición conceptual para partir de la lectura de lugar

\begin{tabular}{|l|l|l|}
\hline \multicolumn{1}{|c|}{ Obra } & \multicolumn{1}{c|}{ Localización } & \multicolumn{1}{c|}{ Concepto } \\
\hline $\begin{array}{l}\text { Piscinas das Marés, } \\
\text { (1961-1966) }\end{array}$ & $\begin{array}{l}\text { Matosinhos, Gran } \\
\text { Oporto, Portugal. }\end{array}$ & $\begin{array}{l}\text { Aprovechar la existencia de grandes ma- } \\
\text { cizos rocosos entre los cuales crear muros } \\
\text { de contención para delimitar las piscinas, } \\
\text { potenciando así los espacios naturalmen- } \\
\text { te conformados y aprovechables para } \\
\text { crear los embalses. }\end{array}$ \\
\hline $\begin{array}{l}\text { Restaurante Boa } \\
\text { ra, (1963) }\end{array}$ & $\begin{array}{l}\text { Matosinhos, Gran } \\
\text { Oporto, Portugal. }\end{array}$ & $\begin{array}{l}\text { Utilizar la concavidad natural formada en } \\
\text { el montículo para ubicar el edificio, el cual } \\
\text { oculta sus muros entre piedras y resalta } \\
\text { la cubierta de teja como el elemento } \\
\text { formalmente predominante, su cúspide } \\
\text { parece cerrar el perfil de este pequeño } \\
\text { cerro rocoso. }\end{array}$ \\
\hline $\begin{array}{l}\text { Escuela de Arqui- } \\
\text { tectura de Oporto, } \\
\text { (1985-1996) }\end{array}$ & Oporto, Portugal. & $\begin{array}{l}\text { Fragmentar formalmente el conjunto para } \\
\text { abrir desde su núcleo y desde los edificios } \\
\text { las vistas hacia el paisaje del estuario del } \\
\text { río Duero. }\end{array}$ \\
\hline $\begin{array}{l}\text { Centro Gallego de } \\
\text { Arte Contemporáneo, } \\
\text { CGAC, (1993) }\end{array}$ & $\begin{array}{l}\text { Santiago de Compos- } \\
\text { tela, Galicia, España }\end{array}$ & $\begin{array}{l}\text { Adaptación a la forma triangular del } \\
\text { terreno, crear un atrio interior también } \\
\text { triangular a partir del cual se desarrolla } \\
\text { espacialmente el inmueble. }\end{array}$ \\
\hline
\end{tabular}

\section{CONCLUSIÓN}

El concepto arquitectónico es la idea creativa a partir de la cual se va generando en el proceso de composición el desarrollo de un proyecto, y en el caso particular de Álvaro Siza, esta se desarrolla en función de la lectura del lugar en donde se situará el nuevo edificio. La premisa del arquitecto portugués suele ser: "los arquitectos no inventamos nada, solo transformamos la realidad" (Baborski, 2001, p.262), lo cual aduce al papel preponderante que asigna al contexto existente y a cómo, a partir de la lectura de este, suelen surgir las claves que permiten formular y robustecer un concepto arquitectónico. Los elementos fundamentales de esta lectura son la comprensión de la naturaleza del lugar, su clima y las soluciones empíricas que la arquitectura le antepone como un medio de integración; la compatibilidad del edificio con las actividades y la escala preexistente, la interpretación de los límites, el confinamiento del espacio y muy particularmente el carácter o personalidad de un lugar de acuerdo con su conformación paisajística, singularidad histórica, simbólica y social. 
Aunque parezca obvio lo antes mencionado, el vínculo entre concepto arquitectónico y lugar actualmente se encuentra disociado, debido a la sobrevaloración artística de la forma y el lenguaje plástico en los procesos de diseño. Además, con frecuencia, esta situación es ocasionada por la visión abstracta que se tiene del edificio como un objeto meramente artístico y aislado, cuando en la arquitectura cada lugar que aloja una obra es irrepetible, por lo tanto, cada problema de diseño debe adquirir una dimensión en función de sus características únicas.

Se busca entonces, a partir del presente, invitar a los estudiantes de arquitectura a reflexionar sobre las posibilidades creativas que brinda el lugar del proyecto, a descifrar las claves que entraña cada sitio y a partir de ello definir un partido que busque una continuidad cuando se presentan elementos valiosos paisajísticos naturales o urbanos a preservar. Finalmente, se llega a una serie de puntos específicos planteados como acciones de diseño recurrentes en la valoración de las cuatro obras expuestas: la formación topológica como recurso creativo; la noción de evolución y envejecimiento de un edificio debido a la acción que crea la naturaleza; el predominio visual del paisaje, particularmente del horizonte; la formulación de espacios de transición entre adentro y afuera, entre lo íntimo y lo urbano o paisajístico; el respeto por la escala y morfología preexistente y por los edificios que en el lugar tienen un valor en la memoria colectiva.

\section{REFERENCIAS}

Baborsky, M. (2001). Siglo XX, Arquitectura. Madrid: Electa.

Bonamusa, J. (2011). Oporto. Barcelona: Alhena Media.

Campos, C. (2014). Álvaro Siza Textos. Madrid: Abada.

Carratalá, L. (2003). Álvaro Siza y la arquitectura universitaria. Valencia: PUV, Universidad de Valencia.

Fleck, B. (1999). Álvaro Siza. Madrid: Ediciones Akal. 
Frampton, K. (1985). Hacia un regionalismo crítico: Seis puntos para una arquitectura de resistencia. La posmodernidad, Barcelona: Kairós.

Gómez Luque, M., Gómez Luque, A., y Godoy, G. (2011). 12 Arquitectos contemporáneos. Buenos Aires: Editorial Nobuko.

Illescas A. (2017). Alvaro Siza; lugar y crisis (Tesis doctoral). Universitat Politècnica de Catalunya, Barcelona, España.

Kliczkowski, H. (2001). Álvaro Siza. Madrid: Loft publications.

Muñoz A. (2019). El proyecto de arquitectura: concepto, proceso y representación. Barcelona: Reverté.

The Hyatt Foundation (2021). Pritzker laureates, Alvaro Siza. Reuperdo de https:// www.pritzkerprize.com/laureates/1992 